\title{
De las unidades de cuidado coronario a las unidades de cuidado intensivo cardiovascular: una evolución inevitable
}

\author{
Dr. José A. Pérez Arrarte
}

Una perspectiva histórica

El concepto de las unidades de cuidado intensivo cardiovascular (UCIC) proviene de lo que en principio se denominó unidades de cuidado coronario (UCC). Las descripciones iniciales se deben a Desmond Julian, desde su trabajo en la Royal Infirmary en Edimburgo en el Reino Unido en 1961 ${ }^{(1)}$, y Morris Wilburne en Estados Unidos a través de un trabajo presentado en el meeting de la American Heart Association de $1961^{(2)}$. En sus descripciones iniciales, estas unidades se desarrollaron para el tratamiento del infarto agudo de miocardio (IAM) y basaban su funcionamiento en algunos principios esenciales: a) monitoreo continuo del ritmo cardíaco; b) inicio rápido de reanimación cardiopulmonar y desfibrilación cardíaca; c) personal médico y de enfermería entrenados en dichos pacientes y con el equipamiento necesario en el sitio de guardia. Wilburne destacaba que se requería no solo del reconocimiento adecuado de los patrones clínicos y electrocardiográficos, sino también de la "disponibilidad de un repertorio de actividades bien disciplinadas y bien ensayadas en caso de responder ante un paro cardíaco".

Durante esa primera década de la evolución de las UCC su papel se extendió a través del aporte de Bernard Lown, del tratamiento de las arritmias que complicaban el IAM al intento de prevención de las mismas y a un manejo más temprano e integral de los síndromes coronarios agudos ${ }^{(3)}$. Para el inicio y mediados de la década de 1970, en el mundo industrializado se popularizaron las UCC y pronto se fueron orientando hacia nuevos paradigmas en el manejo y la prevención de la disfunción miocárdica, consecuencia de la enfermedad coronaria. Killip y
Kimball(4), así como Forrester y Diamond ${ }^{(5)}$ introdujeron categorías clínicas y hemodinámicas que contribuyeron a discriminar grupos de pacientes con IAM y precisar mejor el manejo y el pronóstico de los mismos.

El desarrollo inicial de las UCC modificó el pronóstico del IAM, desde un nivel de mortalidad hospitalaria en el entorno de $30 \%-40 \%$ a fines de la década de 1950 y principios de los años 60 , a cerca de la mitad de ese valor a fines de los años 70(6). Para valorar el impacto de este logro se debe tomar en cuenta que por esos años el IAM tenía una mayor incidencia y afectaba a un mayor número de jóvenes que en la actualidad, pues la calidad de la prevención de la enfermedad cardiovascular era primitiva, con mayor prevalencia de tabaquismo y peor calidad del control de los demás factores de riesgo.

En Uruguay, a fines de la década de 1970 los IAM comienzan a ser ingresados en unidades de cuidados intensivos polivalentes y a inicios de los años 80 se crean las primeras UCC específicas, asistiendo un volumen suficiente de pacientes para obtener los beneficios que había demostrado la experiencia mundial ${ }^{(7)}$. Hasta ese entonces muchos pacientes con síndromes coronarios agudos eran ingresados en salas de hospitalización general o, en los casos de menor gravedad, eran asistidos en domicilio.

\section{Reperfusión miocárdica: la revolución de} los años 80

En la década de 1980 surge como un nuevo paradigma el concepto de reperfusión miocárdica al obtener la desobstrucción de la arteria coronaria culpable 
del IAM, inicialmente a través de terapia trombolítica (afirmada en grandes estudios multicéntricos encabezados por el GISSI) $)^{(8)}$ y luego a través de las intervenciones con catéter que impulsaron el desarrollo de lo que hoy conocemos como cardiología intervencionista. Los tratamientos de reperfusión miocárdica obtuvieron un beneficio adicional sobre la mortalidad del IAM, que la redujo nuevamente casi a la mitad del nivel obtenido a fines de los años $70^{(9)}$. El desarrollo de estas tecnologías también mejoró los resultados clínicos en todo el espectro de la enfermedad coronaria aguda.

Si bien los resultados iniciales obtenidos en el tratamiento del IAM entusiasmaron a la comunidad médica, el desarrollo de UCC especializadas en nuestro país fue lento. Salvo la pionera UCIP del Hospital Español (fundada en 1980) y la unidad coronaria del Hospital Militar, hacia los años 90, se desarrollaron unidades de cuidados críticos cardiovasculares únicamente en instituciones que poseían centros de hemodinamia y cirugía cardíaca y no fue sino hasta bien entrada la década del 2000 que se creó la del Hospital de Clíni$\operatorname{cas}^{(7)}$.

Con el paso del tiempo las UCC admitieron un mayor número de pacientes abarcando un espectro más amplio de la patología cardiovascular, en tanto se fueron desarrollando nuevas estrategias de tratamiento e incorporando nuevos recursos técnicos y procedimientos. La disminución de la mortalidad cardiovascular, y en particular de los eventos coronarios agudos, ha dado lugar a una importante población de edad avanzada con enfermedad cardiovascular crónica, multiintervenida, y con numerosas comorbilidades. Estos pacientes complejos y vulnerables tienen mayor frecuencia de hospitalización y el manejo de sus descompensaciones ha requerido una atención cada vez más especializada tanto a nivel convencional como intensivo cardiovascular.

Por otra parte, el avance en los procedimientos de reperfusión en el IAM no solo ha mejorado el pronóstico de esta condición, sino que paradójicamente también ha hecho mucho más sencillo el manejo de los pacientes que fueron el objeto inicial de las UCC, que actualmente en la gran mayoría de los casos pueden manejarse en áreas de cuidado intermedio y tienen una breve estancia hospitalaria.

De modo que una conjunción de factores modificaron el perfil de los pacientes hospitalizados por enfermedad cardiovascular aguda y ha hecho que las primigenias UCC, devotas del cuidado de una población selecta de pacientes con síndromes coronarios agudos, evolucionaran a UCIC que atienden a una población de mayor edad, con patologías más variadas y complejas. Esta evolución implica además un aumento sostenido de costos.

\section{Nuevas exigencias}

En la actualidad los especialistas en medicina cardiovascular que se desempeñan en unidades de cuidado intensivo cardiovascular han debido ampliar su manejo a todo el espectro de la especialidad, lo que incluye: A) El tratamiento de la insuficiencia cardíaca avanzada, sistemas de monitoreo invasivo y dispositivos de soporte circulatorio. B) Manejo de disfunción valvular aguda nativa o protésica. C) Tratamiento de la hipertensión pulmonar y de cardiopatías congénitas en el adulto. D) Cuidado del paciente con arritmia, marcapasos, resincronizadores y cardiodesfibriladores implantados. F) Manejo de métodos de imagen que permiten orientar el tratamiento al pie de la cama.

Ello implica, además, la interrelación con el equipo de cardiología general en lo que refiere a las decisiones estratégicas y de medio plazo que hacen a la continuidad de la asistencia, estrategias de prevención, así como a la interrelación con los subespecialistas (cirujanos, hemodinamistas, arritmólogos) $)^{(10)}$.

Las múltiples comorbilidades del paciente cardiovascular de edad avanzada, en particular la diabetes, la insuficiencia renal y la enfermedad pulmonar obstructiva, exigen destrezas de manejo en áreas superpuestas con otras especialidades.

Este panorama genera también una importante superposición entre las áreas de cuidados cardiovasculares y las áreas de terapia intensiva general cuyos límites se han difuminado, en particular en el manejo de la falla multiorgánica y el paciente séptico, terrenos donde el manejo tiende a ser cada vez más normatizado (infección hospitalaria, pautas de sedación, nutrición, etcétera).

Esta mayor complejidad del paciente con patología cardiovascular aguda no debe quitar especificidad a las UCIC, pues ello no resulta en una mejoría de la asistencia. Las principales sociedades científicas en el mundo han orientado estos problemas de los límites de cada especialidad, y la interrelación entre las mismas, hacia la colaboración y no hacia la exclusión o la conquista de nichos de protección laboral como se observa con frecuencia.

\section{Problemas de organización}

Algunos dilemas del pasado respecto a las características de la organización y el equipo médico en el área de cuidado intensivo cardiovascular parecen estar actualmente laudados. En los países del pri- 
mer mundo, principalmente en Estados Unidos, han coexistido dos modelos de organización de los centros de terapia intensiva, los de tipo "abierto" y los de tipo "cerrado". El servicio es "abierto" cuando el paciente es atendido por su médico (internista o cardiólogo), quien toma las decisiones sin pertenecer al staff del centro, el que eventualmente puede ofrecer asesoramiento en terapia intensiva o en la especialidad que el médico tratante solicite. Ese médico puede llevar a cabo todos los pasos de la asistencia. Hasta hace pocos años este tipo de organización era la regla. En cambio, las unidades de tipo "cerrado" están dotadas de un equipo médico estable que toma la gran mayoría de las decisiones, más allá de un mayor o menor grado de interacción con el médico tratante. Existe un cuerpo de evidencia importante que favorece al modelo "cerrado", con un equipo médico presente en régimen de 24 horas, en términos de resultados (incluyendo mortalidad), lo que es aplicable tanto a las unidades generales como a las especializadas en el área cardiovascular ${ }^{(11)}$. Es interesante que esta discusión no se aplicaría en Uruguay, y probablemente en otros países de Latinoamérica, dado que en la mayoría de ellos las unidades de cuidados intensivos se desarrollaron con un modelo "cerrado" desde su origen.

En los últimos años, varias sociedades científicas de primera importancia (Reino Unido, Estados Unidos, Canadá, Sociedad Europea de Cardiología [ESC]) han planteado esquemas de organización para las UCIC en tres niveles de complejidad, adoptando una escala tomada de los centros de trau$\mathrm{ma}^{(12-15)}$.

El nivel 1 es el más complejo y comprende centros de tratamiento intensivo que asumen la responsabilidad por los procedimientos de máxima complejidad y de máximo costo. Típicamente estos centros se ubican en instituciones de nivel terciario con disponibilidad de cardiología intervencionista y cirugía cardíaca, y deben incluir capacidad de manejo pre y postrasplante cardíaco, asistencia circulatoria mecánica, asistencia respiratoria avanzada e hipotermia controlada. Estos centros están dirigidos por cardiólogos con formación en terapia intensiva o coliderados con especialistas en medicina intensiva; deben contar con presencia de médicos especialistas las 24 horas, una relación de enfermería-paciente 1:1 y recursos de formación para residentes, fellows y personal de enfermería.

Los centros de nivel 2, a diferencia de los anteriores, no cuentan con asistencia circulatoria mecánica o como máximo disponen de contrapulsación aórtica (técnica que se utiliza cada vez menos). Dichas unidades idealmente cuentan con presencia de médicos con formación en cardiología y terapia in- tensiva durante las 24 horas, y una relación de enfermería-paciente que tiende a $1: 1$, pero no obligatoriamente. Pueden compartir el área física con camas de cuidado intensivo médico o quirúrgico general.

Los centros de nivel 3, si bien pueden tener el carácter y especificidad de un área de cuidado intensivo cardiovascular, pueden coexistir con un área de cuidado intensivo general o con un área de cuidado intermedio. Las mismas disponen de recursos de asistencia respiratoria mecánica, tratan la insuficiencia cardíaca severa con drogas vasoactivas, pero no realizan monitoreo hemodinámico invasivo ni pueden manejar el shock cardiogénico. Pueden tratar el síndrome coronario agudo y las arritmias más comunes utilizando procedimientos con un grado de complejidad correspondiente a un hospital de nivel local o regional.

\section{Formación curricular}

Si bien en la literatura científica hay detallada discusión sobre estos temas organizacionales, no se cuestiona la importancia de la existencia de UCIC con médicos cardiólogos participando activamente de la organización y operación de estos centros y se establecen variados esquemas de formación curricular de acuerdo con las diferentes organizaciones y medios académicos ${ }^{(11)}$. Existe más de un camino para obtener una especialización que contemple la formación en cardiología y en cuidado intensivo, lo que también dependerá de la comunidad médica que se trate y para qué tipo de complejidad de UCIC. Si bien en los países del primer mundo se establece un marco curricular bastante riguroso para la formación de los especialistas en cardiología intensiva, hay que destacar que ya se contempla una importante escasez de recursos humanos y económicos para cubrir las necesidades actuales. Esto también concierne al personal de enfermería, nutricionistas, fisioterapeutas, laboratoristas, así como ecónomos y especialistas en informática(16).

En Estados Unidos el requerimiento de cardiólogos con destrezas en cuidado intensivo y, a la inversa, de intensivistas con competencias en cuidado cardiovascular, ha llevado a las autoridades del American College of Cardiology a desarrollar programas de formación que superen las carencias de ambas especialidades. Esto se ha canalizado a través del COCATS 4 (Core Cardiovascular Training Statement), un organismo encargado de supervisar la estructura curricular de los programas de especialización en cardiología, que desde 2008 ha realizado recomendaciones específicas para el área de cuidados cardiovasculares críticos ${ }^{(17)}$. 
Los programas actuales definen tres niveles de intensidad y duración diferentes que otorgan capacidad para desarrollar tareas en escenarios de mayor o menor complejidad.

El nivel 1 es el más básico, requiere formación completa en cardiología y una rotación por el área de cuidado intensivo no inferior a ocho semanas, para exponerse al manejo de los problemas más habituales del área, entre los que se incluyen: complicaciones de los síndromes isquémicos agudos, insuficiencia cardíaca avanzada, disfunción valvular, hipertensión severa y patología aórtica aguda, trastornos severos de la circulación pulmonar, arritmias y complicaciones de la enfermedad pericárdica. Este nivel está pensado para capacitar a aquellos cardiólogos que deben resolver consultas cardiológicas en áreas de cuidado intensivo sin formar parte del staff médico de dicha área. Debe tenerse en cuenta que esta función de cardiólogo consultante es trascendente, pues en medios como en Estados Unidos al menos la mitad de las unidades de cuidado intensivo no están dotadas de médicos intensivistas y la mayor parte de la tarea la conducen médicos visitantes (régimen "abierto").

En el nivel 2 el médico desarrolla su programa completo de aprendizaje en cardiología conjuntamente con rotaciones y capacitación en terapia intensiva durante los tres años de entrenamiento (período de duración habitual de los fellowships en cardiología en Estados Unidos y en gran parte de los países que participan de la ESC). En dicho nivel además del contacto con las condiciones cardiovasculares críticas, los médicos adquieren destrezas completas en monitoreo no invasivo e invasivo, incluyendo las herramientas propias del estudio hemodinámico. Quienes se entrenan a este nivel comparten e incluso pueden liderar el staff médico de la UCIC.

En el nivel 3, a la formación completa en medicina cardiovascular, se suma el aprendizaje en terapia intensiva acorde con las demandas de las unidades de cuidado intensivo de nivel 1 , o sea centros de cuidado intensivo con capacidad de asistencia respiratoria mecánica, asistencia circulatoria, posoperatorio de cirugía cardíaca, monitoreo invasivo y no invasivo incluyendo métodos de imágenes, así como capacidad de liderazgo o coparticipacion con intensivistas y cardiólogos subespecialistas y también manejo de herramientas de gestión e investigación.

Hasta hace poco tiempo en Estados Unidos, Europa y en hospitales de alto nivel en Latinoamérica muchos cardiólogos e intensivistas con formación de nivel 3 procedían de haber completado curricularmente ambas especialidades. Actualmente se están desarrollando programas de especialidad en cardiología específicamente para el aprendizaje de cuidado intensivo con el agregado de un año más a los tres años de especialización, otorgando plena proficiencia en cuidado intensivo cardiovascular.

La ESC, a través de un grupo de trabajo de la Acute Cardiac Care Association, ha definido desde 2014 una línea curricular para la adquisición de las competencias apropiadas para aquellos cardiólogos dedicados al cuidado intensivo cardiovascular. Similar al COCATS 4, ha definido niveles de entrenamiento entre 1 y 3 , y esta última categoría se adquiere a través de un año adicional de entrenamiento en cuidado intensivo más allá de la formación estándar en cardiología. Notoriamente, la ESC reconoce disparidades nacionales y regionales en el desarrollo de las UCIC, por lo que flexibiliza la adquisición de ciertas capacidades a la disponibilidad de posibilidades y recursos de los diferentes centros en los diferentes países y regiones ${ }^{(12,14)}$.

En nuestro medio ya fue planteado en el pasado, y debe volver a plantearse, que los cardiólogos en formación deben tomar contacto con las unidades de terapia intensiva y sobre todo con las áreas existentes con dedicación a pacientes cardiovasculares, para que de este modo se formen recursos humanos cada vez más necesarios. De lo contrario, la ausencia de médicos, ya sea intensivistas con sólida formación en medicina cardiovascular así como de cardiólogos con formación en cuidado intensivo, dificultará la expansión y sostenibilidad de verdaderas UCIC. Debemos seguir insistiendo en la necesidad del trabajo colaborativo entre cardiólogos e intensivistas tanto a nivel de las instancias de aprendizaje formal a nivel universitario, como en el ámbito asistencial público y privado. Es necesario que aquellos médicos en formación con interés en el cuidado intensivo cardiovascular encuentren un panorama bien definido desde el punto de vista curricular, y que las instituciones educativas, a su vez, definan las competencias adecuadas para nuestro nivel de asistencia, de modo de poder alcanzar algo próximo a la certificación en cuidado intensivo cardiovascular.

\section{A modo de conclusión}

Conviene recordar algunas recomendaciones finales del boletín editado recientemente por el Grupo de Trabajo en Cuidado Cardiovascular Agudo de la British Society of Cardiology ${ }^{(13)}$ :

- Los pacientes que se presentan con condiciones cardíacas tienen demostradamente mejor pronóstico si son tratados en áreas especializadas en cardiología.

- Una significativa proporción de estos pacientes no son manejados en servicios de cardiología conduciendo a mayor morbilidad y mortalidad y a mayores costos para el Servicio Nacional de Salud. 
- Los pacientes que se presentan con una condición cardíaca aguda deben ser manejados por un equipo cardiológico especializado, multidisciplinario, con acceso a las claves de diagnóstico y tratamiento toda vez que sea necesario.

- Todo hospital que admita a pacientes médicos agudos debe tener una unidad de cuidado cardíaco agudo, con staff y equipamiento adecuado, donde deberán ser manejados los pacientes de alto riesgo con un diagnóstico cardíaco primario.

- Todos los pacientes de alto riesgo cardíaco deben tener acceso a una UCIC y el acceso no debe restringirse solo a los pacientes con síndrome coronario agudo.

\section{Agradecimientos}

El autor agradece la colaboración del Consejo Editorial de la Revista Uruguaya de Cardiología en la revisión y corrección de este artículo.

\section{Bibliografía}

1. Julian DG. Treatment of cardiac arrest in acute myocardial ischaemia and infarction. Lancet 1961;2 (2707):840-4.

2. Wilburne M. Coronary care unit: new approach to treatment of acute coronary occlusion. Circulation 1961; 24:1071.

3. Lown B, Fakhro AM, Hood WB Jr, Thorn GW. The coronary care unit: new perspectives and directions. JAMA 1967; 199(3):188-98.

4. Killip T 3rd, Kimball JT. Treatment of myocardial infarction in a coronary care unit: a two year experience with 250 patients. Am J Cardiol 1967; 20(4):457-64.

5. Forrester JS, Diamond G, Chatterjee K, Swan HJ. Medical therapy of acute myocardial infarction by application of hemodynamic subsets (first of two parts). N Engl J Med 1976; 295(24):1356-62. doi: 10.1056/NEJM197612092952406

6. Fye WB. Resuscitating a Circulation abstract to celebrate the 50 anniversary of the coronary care unit concept. Circulation 2011;124(17):1886-93. doi: 10.1161/CIRCULATIONAHA.111.033597

7. Sandoya E. Cardiopatía isquémica aguda y la revolución en cardiología. Rev Urug Cardiol 2013;28(3): 399-402.
8. Rovelli F. Effectiveness of intravenous thrombolytic treatment in acute myocardial infarction. Gruppo Italiano per lo Studio della Streptochinasi nell'Infarto Miocardico (GISSI). Lancet 1986;1(8478): 397-402. En PubMed sin lista de autores

9. Brauwald E. Evolution of the management of acute myocardial infarction: a 20th century saga. Lancet 1998;352:1771-4.

10. Bourke ME. Coronary Care Unit to Cardiac Intensive Care Unit: Acute Medical Cardiac Care Adapting With the Times. Can J Cardiol 2016;32(10): 1197-9. doi: 10.1016/j.cjca.2016.02.001

11. Hill T, Means G, van Diepen S, Paul T, Katz JN. Cardiovascular critical care: a perceived deficiency among US trainees. Crit Care Med 2015;43(9):1853-8. doi: 10.1097/CCM.0000000000001074.

12. Katz JN, Minder M, Olenchock B, Price S, Goldfarb M, Washam JB, et al. The genesis, maturation, and future of critical care cardiology. J Am Coll Cardiol 2016;68 (1): 67-79. doi: 10.1016/j.jacc.2016.04.036.

13. Walker DM, West NE, Ray SG. From coronary care unit to acute cardiac care unit: the evolving role of specialist cardiac care. Heart 2012;98(5):350-2. doi: 10.1136/heartjnl-2011-301459

14. Hasin J, Danchin N, Filippatos G, Heras M, Janssens U, Leor J, et al. Recommendations for the structure, organization, and operation of intensive cardiac care units. Eur Heart J 2005; 26(16):1676-82.

15. LeMay M, van Diepen S, Liszkowski M, Schnell G, Tanguay JF, Granger CB, et al. From Coronary Care Units to Cardiac Intensive Care Units: recommendations for organizational, staffing, and educational transformation. Can J Cardiol 2016;32:(10) 1204-13. doi: 10.1016/j.cjca.2015.11.021

16. Morrow DA, Fang JC, Fintel DJ, Granger CB, Katz JN, Kushner FG, et al. Evolution of critical care cardiology: Transformation of the cardiovascular intensive care unit and the emerging need for new medical staffing and training models: a scientific statement from the American Heart Association. Circulation 2012;126(11):1408-28

17. Halperin JL, Williams ES, Fuster V. COCATS 4 introduction. J Am Coll Cardiol 2015;65(17):1724-33. doi: 10.1016/j.jacc.2015.03.020. 